\title{
Optimized tracking for cooperative sensor systems in multipath environments
}

\author{
D. Schwarz ${ }^{1}$, R. H. Rasshofer ${ }^{1}$, and E. M. Biebl ${ }^{2}$ \\ ${ }^{1}$ BMW Group Research and Technology, Munich, Germany \\ ${ }^{2}$ Fachgebiet Höchstfrequenztechnik, Technische Universität München, Germany
}

\begin{abstract}
In a cooperative sensor system for pedestrian protection, a pedestrian and other road users exchange data by means of radio frequency communication. In the proposed system, the pedestrian carries a transponder which is interrogated by a vehicle and sends an anonymous identification (ID) sequence. By decoding the ID, the interrogation unit in the vehicle detects the presence of the transponder. Evaluating the incident wave of the transponder's answer, a localisation is possible.

In the proposed localization system, the measurement results can be distorted by multipath propagation. Multipath errors result if signals of the same transponder arrive simultaneously at the receiver unit from different directions. In this case, erroneous distances and angles are measured. Because the signals arriving from different directions contain the same transponder ID, they can be assigned to their origin. One of the challenges in post-processing for signal improvement is enhancing the selection of the correct position information by making assumptions about the pedestrian's movement and by knowing the vehicle's current driving parameters. Additionally, information contained in multipath signals is used to form a better estimate for the true position of the transponder. To overcome the problems related to multipath propagation effects, a method is proposed that estimates the origin of a multipath signal and maps the distorted position information back to the true position. A fusion of tracked direct positions and mapped multipath signals leads to an improvement in positioning accuracy.
\end{abstract}

\section{Introduction}

Pedestrian detection approaches based on lidar (Fürstenberg, 2004), radar, or vision-based systems (Gavrila et al., 2004) offer highly accurate positioning information of objects or persons in the vehicle's surroundings. The reliability of these

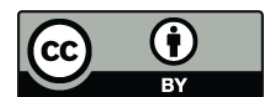

Correspondence to: D. Schwarz

(Daniel.Schwarz@bmw.de) systems has been improved rapidly using new detection, classification and fusion algorithms (Paraschoudis et al., 2006). Current systems are already capable to cover a large number of typical accident scenarios. In scenarios where pedestrians are fully or partially hidden to these sensors either their detection or their correct classification might fail. The results of accident studies show that $40 \%$ of all fatal pedestrian accidents in Germany are caused by a late recognition of the situation by the driver (German In-Depth-Accident Study, 2005). In these cases, a cooperative sensor system could support active safety systems with valuable information. A pedestrian that is occluded to the view of the driver and sensors built in the vehicle can be detected earlier which provides more time for triggering collision avoidance and collision mitigation systems.

\subsection{Related work}

In Shi et al. (2005) a proposed cooperative system determines the DoA of an incident transponder signal. The distance is not measured directly but through triangulation using the detected DoAs at two subsequent locations while the vehicle is moving.

Meinken et al. (2007) describe a cooperative system for traffic safety based on communication and sensor technologies. The concept relies on interactions between an invehicle module and a user's device. Positioning is done by two communication-based distance measurements between the rear-view mirrors and the transponder (Sikora, 2006). Through triangulation the position can be calculated.

Other previous work that mention cooperative systems use infrastructure devices as repeaters (Kubota et al., 2006) but do not use a localisation system integrated into a vehicle.

\subsection{Content}

First, the integrated localisation system in the test vehicle is introduced and measurement principles are described in more detail. The influences of multipath propagation on measurement results are pointed out in Sect. 3. To overcome the drawbacks caused by multipath effects, requirements on

Published by Copernicus Publications on behalf of the URSI Landesausschuss in der Bundesrepublik Deutschland e.V. 

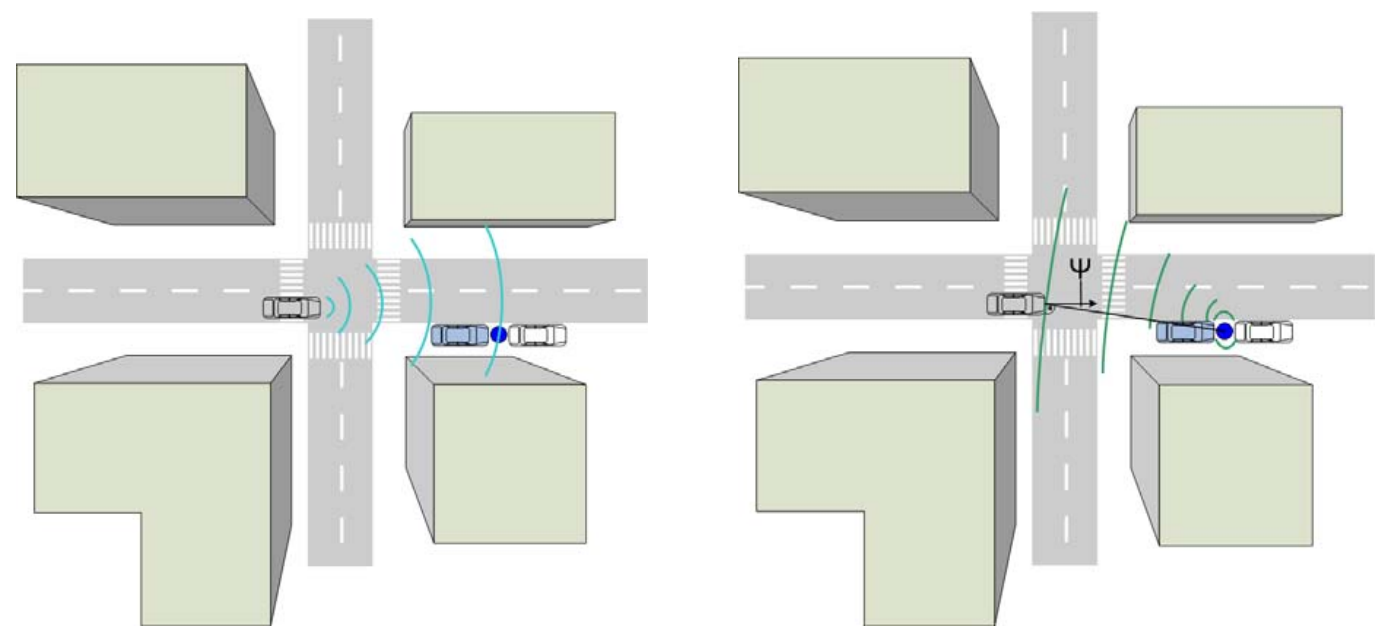

Fig. 1. Interrogation pulse of vehicle in an urban scenario (left), answer pulse of a transponder hidden to the driver between parked cars.

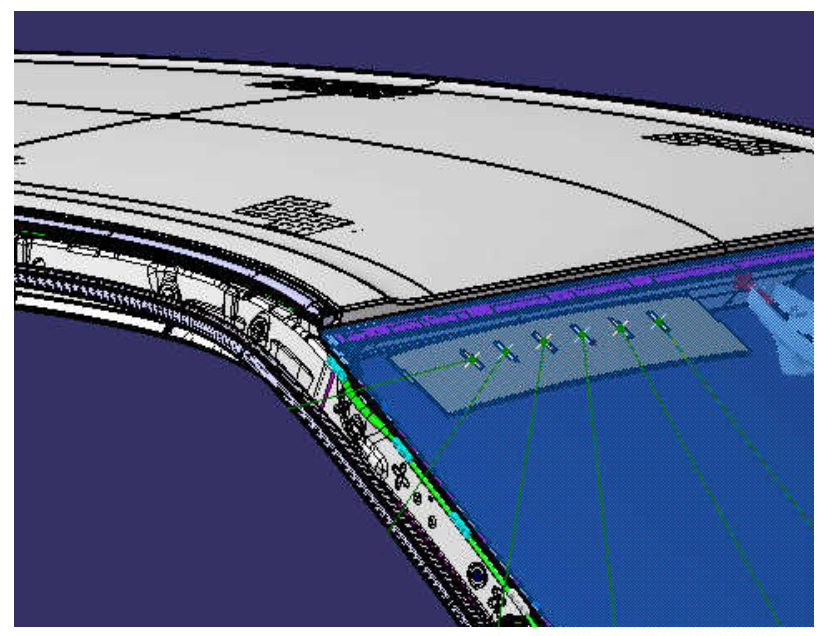

Fig. 2. 3-D model of antenna array integrated behind the front window of test vehicle.

a robust tracking algorithm are deduced, and in Sect. 4 an example for an algorithm which is able to deal with multipath effects is introduced. An outlook on further work and a conclusion are presented in the final sections.

\section{System overview}

\subsection{On board unit}

A central part of the system is an antenna array which is integrated behind the wind shield of the vehicle. The antenna array is used to send an interrogation pulse and to receive the answer from the transponder to be localized. Data samples of the single antenna elements are used for the detection of an object's presence and determination of the object position.

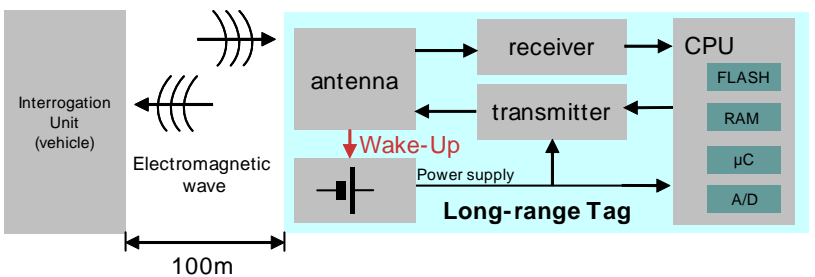

Fig. 3. Interaction between interrogation unit and transponder.

The driver's field of view is not affected by the integrated antennas and no modifications need to be made to the car body or design of the test vehicle (see Fig. 2).

The initial design of the system will utilize ISM bands to ease international licensing. As each ISM band offers different channel properties, three different ISM bands have been experimentally investigated $(434 \mathrm{MHz}, 868 \mathrm{MHz}$ and $2.4 \mathrm{GHz}$ ). Communication with a transponder being optically hidden behind a car represents one of the most challenging scenarios. With all three frequencies the received signal strength from a transponder showed to be strong enough for a reliable detection, even in worst case scenarios. To minimize the antenna array's size, $2.4 \mathrm{GHz}$ has been chosen as operating frequency. Switching to the IEEE $802.11 \mathrm{p}$ standard at $5.9 \mathrm{GHz}$, intended for Car-to-Car $(\mathrm{C} 2 \mathrm{C})$ communication, even would lead to a further reduction of the antenna size.

\subsubsection{Direction-of-Arrival (DoA)}

Using six antennas in the antenna array, an estimation of the DoA can be obtained. If a valid signal sent by a transponder is identified, the amplitudes and phases of the antenna voltages are sampled and used to derive the DoA. Different well-known high resolution DoA estimation algorithms like ESPRIT (Estimation of Signal Parameters via Rotational 

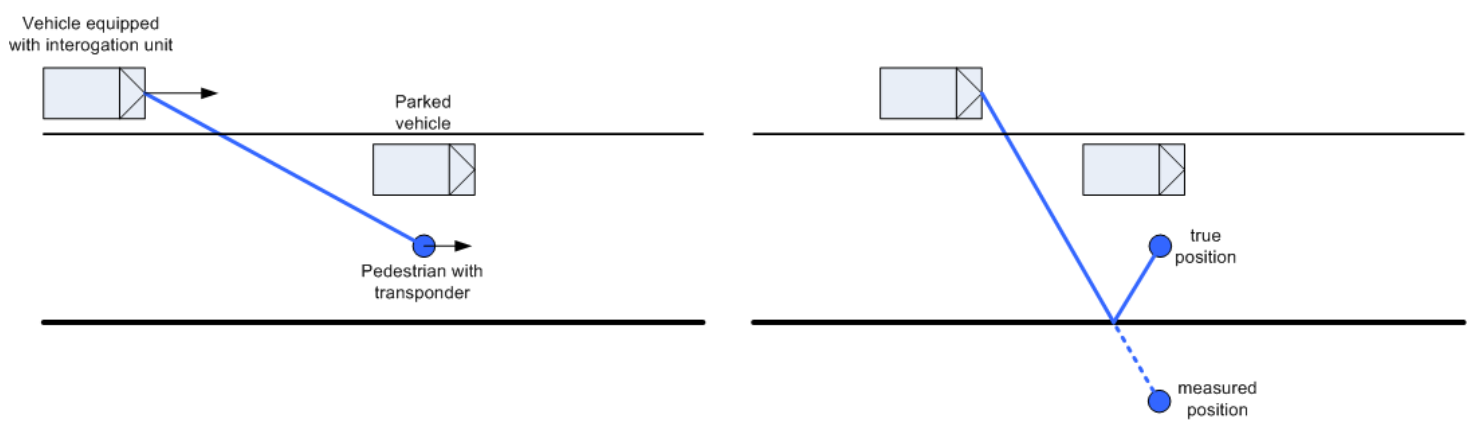

Fig. 4. Correct position of pedestrian is measured (left), multipath signals caused by reflection at a wall lead to different ToF and DoA measurements (right).
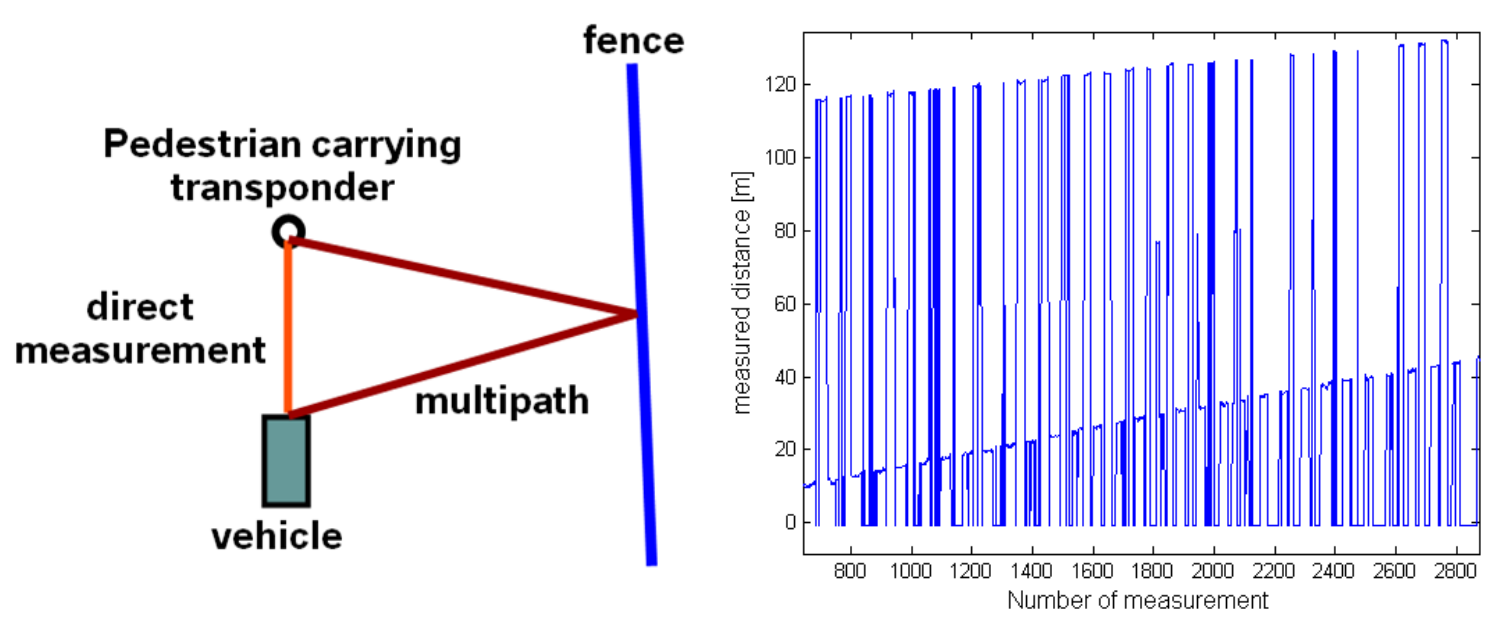

Fig. 5. Measurement setup for cooperative sensor system (left), distance measurement results: multipath propagation causes ambiguity, distance ranges $10-40 \mathrm{~m}$ or $110-130 \mathrm{~m}$ (right).

Invariant Techniques) or MUSIC (Multiple Signal Classification) (Schmidt, 1987) are investigated and applied to the raw data to obtain reliable azimuth angle data. In the first measurements, the transponder's azimuth angle could be estimated with an accuracy of about $1^{\circ}$ in different distances in the case of a Line-of-Sight ( $\mathrm{LoS})$ connection. As expected, the accuracy of the angular measurement decreases without a LoS connection. Tests show the possibility of improving the localisation accuracy, depending on SNR and multipath scenario, by applying suitable tracking algorithms in a postprocessing step.

\subsubsection{Time-of-Flight (ToF)}

The tag's distance can be calculated by a ToF measurement, determining the round trip delay of the interrogation pulse. Furthermore, the change of the measured azimuth angle can be investigated knowing the vehicle's velocity to achieve more accurate positioning (Shi et al., 2005). The ToF measurement is done by means of a subcarrier technique. The received interrogation sequence is decoded and, after a cer- tain time-delay, a response is sent containing information about the time delay and the ID-code (Rasshofer et al., 2007). Knowing the delay time and the time between interrogation pulse and received answer, the distance can be calculated. Since each tag uses a different time-delay, TDMA techniques are applied to allow for separation of the responses from several tags. Without superposition of different responses the precision of both ToF and DoA measurements is enhanced.

\subsection{Transponder}

If the transponder identifies a valid interrogation pulse, it changes into an active state and starts transmitting. This wake up mechanism is suitable to reduce the power consumption and to extend battery lifetime. Another promising approach features the integration of acceleration sensors to shut down the transponder if it is not moved for a longer time and thus is unlikely to be carried by a pedestrian. 


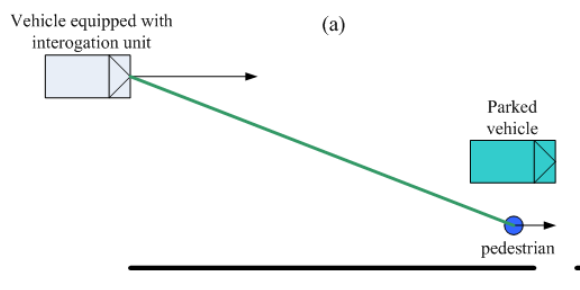

position data in car coordinate system

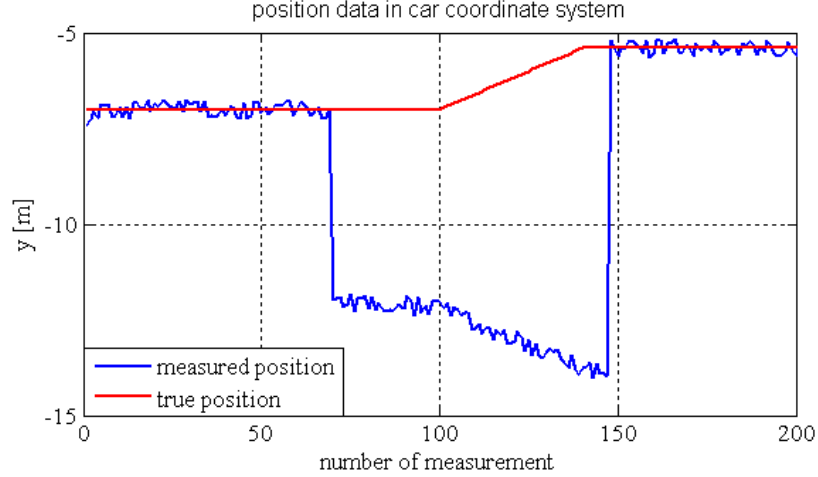

Fig. 7. Simulation data of scenario shown in Fig. 6.

\section{Multipath effects in the cooperative sensor system}

A tracking system for cooperative sensor data needs to deal with multipath propagation of the transponder signals. As sudden strong interferences in the measured position caused by these effects can not be modelled as Gaussian noise, a standard Kalman-Filter (Kalman, 1960) is not the optimal solution for tracking purposes.

In a first approach, the position data corrupted by multipath phenomena are tried to be removed by a tracking procedure. However, measurements showed that there is useful information contained in multipath signals, including information about the moving direction of the transponder, which, if also utilized, can lead to a better positioning accuracy.

\section{Adapted tracking algorithms}

\subsection{Kalman-Filter with plausibility check}

Looking at Fig. 5, a first approach to compensate for multipath effects could be to sort out all measurements influenced by multipath propagation. This is done by a plausibility check of the data when looking at several subsequent measurements. The remaining measurement points are - in this simple case - sufficient for a stable tracking with a Kalman- (b)

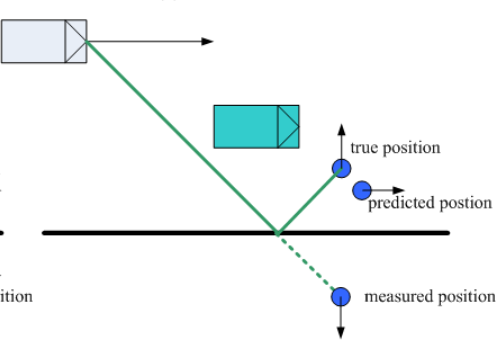

Filter using a system model with constant velocity (CV) for the pedestrian.

However, it can be seen easily that the erroneous measurement values in a range between 110 and $130 \mathrm{~m}$ are increasing similar to the correct measurement points. They indicate a movement of the pedestrian away from the car. The existence of the multipath data in this case can almost completely be explained by a reflection of the transponder signal at a fence near by the measurement setup. As influences on the DoA measurement showed similar results and some interferences could be explained by a simple reflection at planar obstacles, a new approach has been chosen. For further studies and the development of algorithms simulations have been conducted to generate a large number of scenarios.

\subsection{Mapping of multipath signals}

As an example for the various simulations conducted, a setup as shown in Fig. 6 should be considered. A moving vehicle determines the position of a pedestrian moving with a different velocity using the cooperative sensor system. After some simulation time the pedestrian is occluded to the vehicle and due to multipath signals the wrong position is observed in subsequent measurements.

During the occlusion - if multipath signals are neglected only a prediction of the pedestrian's movement can be done by the last estimated velocity vector. Changes in the moving direction can not be recognized in this case. Figure 7 shows position data received in this scenario.

In this approach, a rule-based algorithm detects the sudden change of position data. The position of the pedestrian is predicted using a Kalman-Filter with constant velocity model and for the multipath data, a new track is generated. With the simple assumption that one reflection plane is responsible for the multipath signal, the algorithm tries to calculate the parameters of this plane. As measurements showed, this assumption holds true in many scenarios. The movement of the pedestrian can be predicted quite accurately in the first few hundred milliseconds due to physical limitations like e.g. maximum possible acceleration. If a relation between the predicted position and the tracked multipath position can be found and the reflection plane is observed to be stable for a 

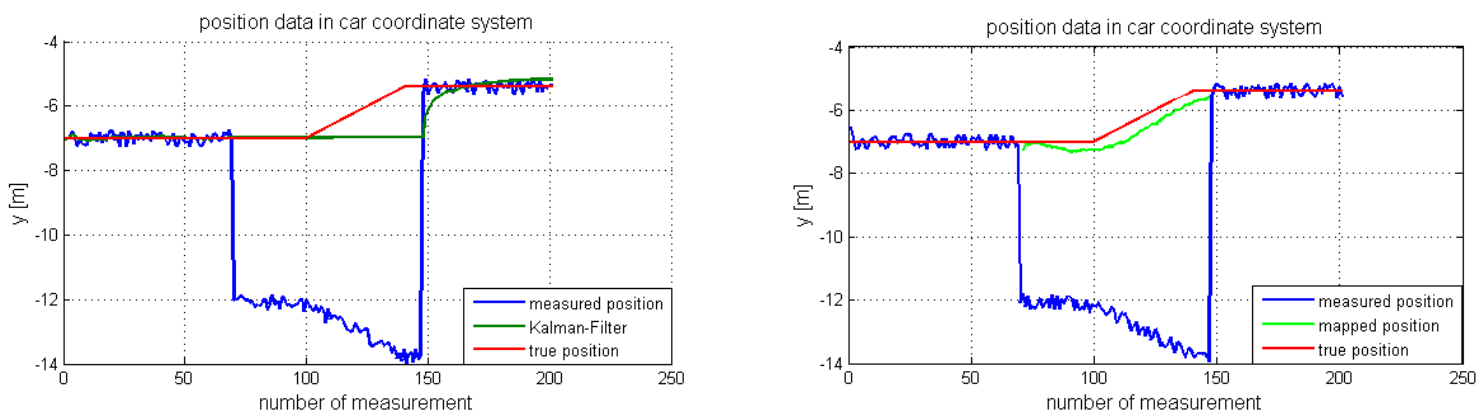

Fig. 8. Comparison between prediction using a Kalman-Filter (left) and the proposed algorithm for multipath data (right).

few cycles, one can start to map back the data to their origin. In the following cycles, the position of the plane in the car coordinate system needs to be updated taking into account the driving parameters of the vehicle, like e.g. velocity or yaw rate. Results are shown in Fig. 8.

This way information, like changes in moving direction, contained in multipath data is interpreted and used for a more robust and stable estimation of the pedestrian's position.

\section{Further work}

Future work should start by adopting algorithmic parameters using measurement data from test scenarios. Increased detection accuracy of a sudden change between a direct signal and a multipath signal should be achieved by taking the received signal strength as additional input.

In the proposed system one position information per cycle was used as input. In the next step, several hypotheses for the position should be used as the measurement principles are able to provide more than one value per cycle. This data can be handled with particle filters based on Sequential Monte Carlo Methods (SMCM) (Doucet et al., 2001) combined with the proposed mapping algorithm.

\section{Conclusions}

A system for pedestrian detection based on cooperative sensor technology has been introduced. The influence of multipath propagation on the system was investigated using ToF and DoA measurement results. A tracking algorithm takes these effects into account and uses a model for the origin of multipath signals. Simulations showed the feasibility of the multipath compensation by mapping erroneous position information back to their origin for an improvement of positioning accuracy.

Acknowledgements. The cooperative sensor system presented in this publication is part of the main efforts in the AMULETT-project which is co-funded by the Bavarian Ministry of Economic Affairs, Infrastructure, Transport and Technology (BayStMWIVT). The project aims to avoid accidents involving vulnerable road users such as pedestrians and bicyclists by means of cooperative sensor technology.

\section{References}

Doucet, A., de Freitas, N., and Gordon, N. (Eds.): Sequential Monte Carlo Methods in Practice, Springer, 2001.

Fürstenberg, K. Ch.: Pedestrian Protection using Laserscanners, IEEE Conference on Intelligent Transportation Systems, 436437, 2005.

Gavrila, D. M., Giebel, J., and Munder, S.: Vision-Based Pedestrian Detection: The PROTECTOR System, IEEE Intelligent Vehicles Symposium, 13-18, 2004.

German In-Depth-Accident Study: http://www.gidas.org, 2005.

Kalman, R. E.: A New Approach to Linear Filtering and Prediction Problems, J. Basic Eng.-T. ASME, 82 (Series D), 35-45, 1960.

Kubota, S., Okamoto, Y., and Oda, H.: Safety Driving Support System Using RFID for Prevention of Pedestrian-involved Accidents, 6th International conference on ITS Telecommunications Proceedings, 226-229, 2006.

Meinken, K., Andreone, L., Guarise, A., and Sikora, A.: WATCHOVER - The Concept Of A Cooperative System For Vehicle To Vulnerable Road Users Communication, 20th International Technical Conference on the Enhanced Safety of Vehicles, 07-0126, 2007.

Paraschoudis, V., Tatschke, T., Walchshaeusl, L., and Lindl, R.: Autonomous braking for collision mitigation purposes by means of multi sensor perception, FISITA World Automotive Congress, F2006V181, 2006.

Rasshofer, R., Schwarz, D., Biebl, E., Morhart, C., Scherf, O., Zecha, S., Grünert, R., and Frühauf, H.: Pedestrian Protection Systems using Cooperative Sensor Technology, Proceedings of the 11th International Forum on Advanced Microsystems for Automotive Applications (AMAA'07), Berlin, 135-145, 2007.

Schmidt, R. O.: Multiple Emitter Location and Signal Parameter Estimation, IEEE T. Antenn. Propag., 34, 276-280, 1987.

Sikora, A.: Vehicle-to-Vulnerable road user cooperative communication and sensing technologies to improve transport safety, D4.1 - Communication technologies specifications, 2006.

Shi, J., Müller, H. C., and Marx, M.: Pedestrian Detection and Localization Using Antenna Array and Sequential Triangulation, IEEE Conference on Intelligent Transportation Systems, Vienna, 126-130, 2005. 